\title{
छs \\ First observations of intensity-dependent effects for transversely split beams during multiturn extraction studies at the CERN Proton Synchrotron
}

\author{
Simone Gilardoni* and Massimo Giovannozzi ${ }^{\dagger}$ \\ CERN, CH 1211 Geneva 23, Switzerland \\ Cédric Hernalsteens ${ }^{*}$ \\ CERN, CH 1211 Geneva 23, Switzerland \\ and EPFL, CH 1015 Lausanne, Switzerland
}

(Received 26 April 2012; revised manuscript received 2 April 2013; published 17 May 2013)

\begin{abstract}
During the commissioning of the CERN Proton Synchrotron multiturn extraction, tests with different beam intensities were performed in order to probe the behavior of resonance crossing in the presence of possible space charge effects. The initial beam intensity before transverse splitting was varied and the properties of the five beamlets obtained by crossing the fourth-order horizontal resonance were studied. A clear dependence of the beamlets' parameters on the total beam intensity was found, which is the first direct observation of intensity-dependent effects for such a peculiar beam type. The experimental results are presented and discussed in detail in this paper.
\end{abstract}

DOI: 10.1103/PhysRevSTAB.16.051001

PACS numbers: $29.20 .-\mathrm{c}, 29.27 . \mathrm{Bd}$

\section{INTRODUCTION}

The multiturn extraction (MTE) [1,2] has been proposed as a new beam manipulation in the transverse plane. The idea is to cross a resonance exhibiting stable islands. The beam will be eventually trapped in the islands as they move through the phase space area occupied by the charged particles. As a result of this manipulation the beam will be split in the transverse plane. In the case of a stable resonance, two structures will be generated: one representing the beam trapped in the islands, with an effective length corresponding to the machine length, times the resonance order; the second, made of the beam left in the center of the phase space, with a length corresponding to a single machine circumference. In the case of unstable resonances, only a single structure with a length corresponding to a multiple (equal to the resonance order) of the machine circumference is generated.

Another important difference between the use of a stable vs unstable resonance is that the latter will automatically generate equally populated structures, while stable resonances do not guarantee that the beam intensity is equally shared among the two disconnected structures. Once the resonance is crossed and the beam split, it is then possible to extract the charged particles over several machine turns, corresponding to the order of the resonance used for the splitting and its stability type.

\footnotetext{
*simone.gilardoni@cern.ch

massimo.giovannozzi@cern.ch

‡cedric.hernalsteens@cern.ch
}

Published by the American Physical Society under the terms of the Creative Commons Attribution 3.0 License. Further distribution of this work must maintain attribution to the author(s) and the published article's title, journal citation, and DOI.
It should be emphasized that such an extraction method is aimed at providing the most uniform filling of a receiving machine with a circumference length different from that of the extracting machine. At CERN, this is the case for the Proton Synchrotron (PS) transfer to the Super Proton Synchrotron (SPS) for fixed target physics. The current extraction method, the so-called continuous transfer [3] is being gradually replaced by MTE in its PS implementation [4] (see Refs. [5,6] for the accounts of the experimental results and Ref. [7] for a report on the commissioning).

The splitting process is performed on the $14 \mathrm{GeV} / c$ extraction flat top. The beam is adiabatically split by varying the horizontal tune during about $\approx 100 \mathrm{~ms}$ from 6.245 to 6.255 . At the same time, two pairs of dedicated sextupoles control the chromaticity and, together with a pair of dedicated octupoles, excite the fourth-order resonance and separate the islands by varying their gradients synchronously with the tune change. These magnets are grouped in two sections each equipped with two sextupoles and one octupole. It is worth recalling that the PS is composed of 100 straight sections and 100 combined function magnets [8] and the two groups of sextupoles and octupoles are located in straight sections 39 and 55. Furthermore, the horizontal beta function reaches its maximum in odd sections and its minimum in even sections (the opposite occurs for the vertical beta function) as can be seen in Fig. 1 where the beta functions and the horizontal dispersion are shown. The MTE elements are located in odd-numbered straight sections to profit from the large horizontal beta function, which enhances the effectiveness of the nonlinear fields. As explained later, a second family of octupoles located in even straight sections is used to reduce the coupling between the horizontal and vertical planes introduced by the nonlinear elements. 


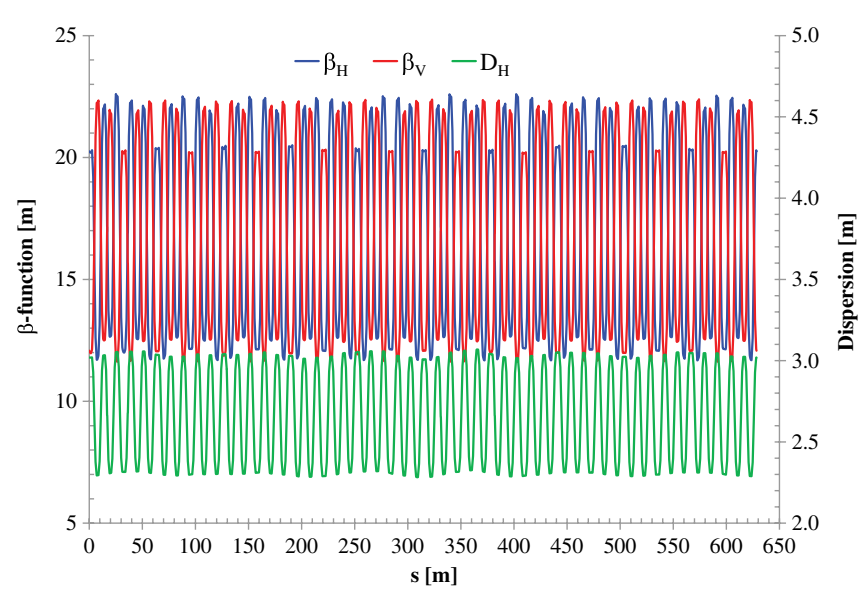

FIG. 1. Overview of the PS optics. The horizontal and vertical beta functions are shown as a function of the distance along the PS circumference, together with the horizontal dispersion. The origin of the horizontal axis is taken in straight section 1 .

The extraction of the beamlets is then performed by means of a series of kicker magnets generating a closed bump of time duration equal to five PS turns, as explained in Ref. [7].

The manipulation of the transverse phase space can be easily generalized to other situations, e.g., it could be used to perform multiturn injection, as described in [9], with some advantages in terms of final value of the beam emittance with respect to the classical multiturn injection. In this framework a first look at space charge effects was made and reported in [10]. Clearly, injection is certainly more prone to space charge effects than extraction due to the difference in energy between the two processes. On the other hand, a first estimate of possible space charge effects for MTE was outlined in [1]. For the CERN-specific application, the typical intensities, and the extraction energy of $14 \mathrm{GeV} / c$, a tune shift due to space charge of the order of a few $10^{-3}$ has been estimated [1]. Hence, while the tune shift is not completely negligible, it is very likely tolerable for the proposed manipulation. During the commissioning stage, although no specific measurement was planned to study intensity-dependent effects for MTE, it was nevertheless possible to probe for the very existence of space charge effects. A first measurement campaign was carried out during the year 2010 run [11], while a second campaign of experimental studies was performed during the year 2012. These measurements represent the core of the discussion reported in the following sections.

The layout of this paper is as follows. In Sec. II the general experimental conditions are described in detail. In Sec. III the experimental results of the first experimental campaign are presented and reviewed, while in Sec. IV a discussion of the possible interpretation of the results is carried out. The results of the second experimental campaign are presented and discussed in detail in Sec. V. Finally, conclusions are summarized in Sec. VI.

\section{EXPERIMENTAL CONFIGURATION}

\section{A. General PS machine setting}

In Fig. 2 the evolution of the main machine parameters as a function of time is shown during the whole magnetic cycle of $1.2 \mathrm{~s}$. The beam is injected at $1.4 \mathrm{GeV}$ (kinetic energy) and extracted at $14 \mathrm{GeV} / c$. At flat top, prior to extraction, dedicated sextupoles and octupoles are powered to generate stable islands, while special quadrupoles are used to cross the fourth-order resonance, as mentioned in the Introduction. An example of the topology of the horizontal phase space is shown in Fig. 3 (left), corresponding to the location of the instrumentation used for the measurements performed in 2010. Initially (upper plot), very small islands are present and the variation of the tune and of the strength of the sextupoles and octupoles is aimed at creating or increasing their size to enhance particle trapping inside the stable islands. At the end of the trapping process (center plot) rather large islands are present at high amplitude, while in the last step (lower plot) the separation between the islands is further increased to prepare for extraction.
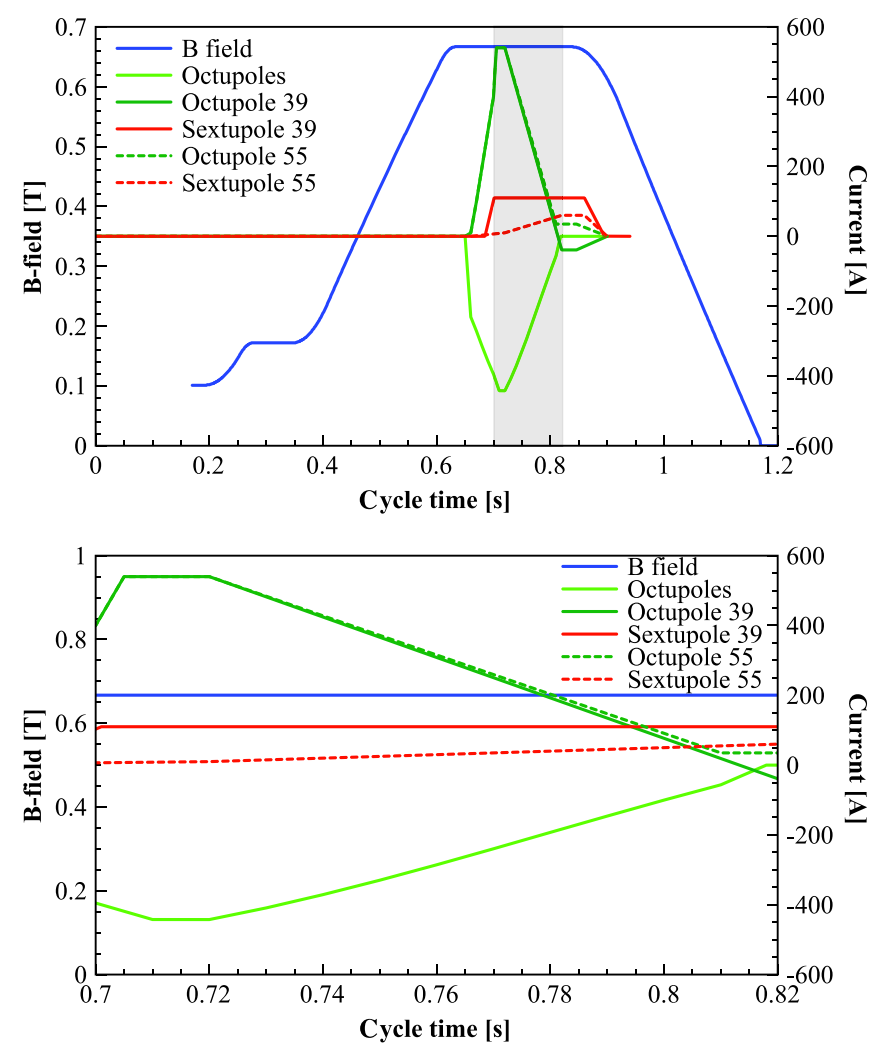

FIG. 2. Overview of the MTE gymnastic (upper). The magnetic cycle is shown together with the evolution of the current for the nonlinear elements used for generating the stable islands and correcting the nonlinear coupling. The shaded area represents the region where the transverse splitting takes place: the closeup of this region is shown in the lower plot. The numbers after the elements indicate the straight section in which they are installed. 

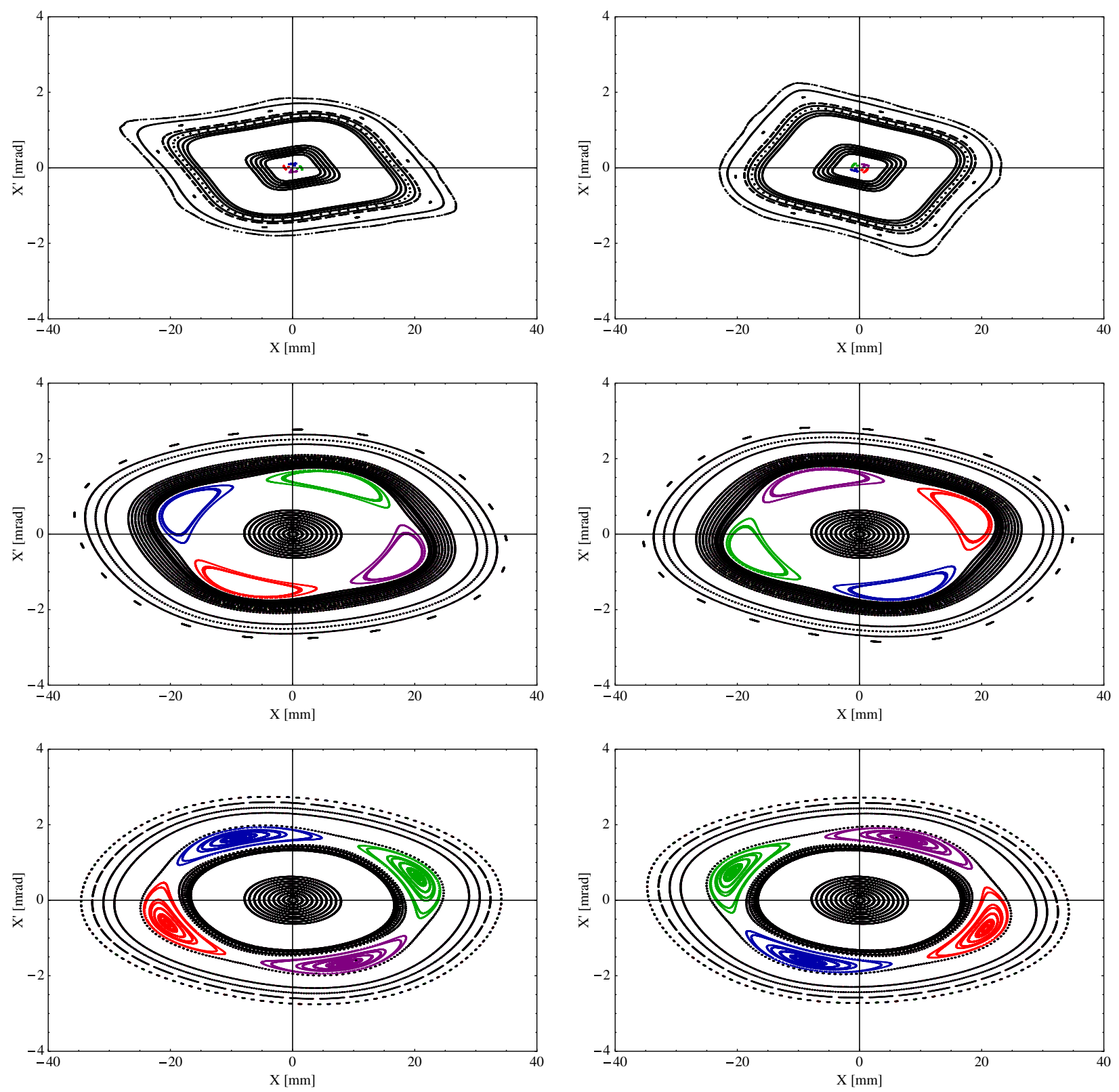

FIG. 3. Overview of the evolution of the phase space topology during the resonance crossing process. The two columns refer to the locations used for the first (left) and the second (right) experimental campaign, respectively. The location of the wire scanner used in 2012 is downstream with respect to the location in 2010. In their path from the upstream to the downstream, section particles in one island move into the island marked with the same color. The upper plot refers to the initial situation at $0.72 \mathrm{~s}$ from the beginning of the magnetic cycle, when islands are very small at the center of phase space. The center plot refers to the situation when the beam is already trapped inside the islands, $0.815 \mathrm{~s}$ from the beginning of the magnetic cycle. The last plot refers to the situation just prior to extraction after $0.835 \mathrm{~s}$ from the beginning of the magnetic cycle. The change in islands' phase between the center and the bottom plot is due to the special circuit of octupoles used to correct the nonlinear coupling term $h_{1,1}$.

The nonlinear magnets introduce an unavoidable nonlinear coupling between the two transverse planes according to [12]

$$
\delta Q_{x}=h_{2,0} \rho_{x}+h_{1,1} \rho_{y}, \quad \delta Q_{y}=h_{1,1} \rho_{x}+h_{0,2} \rho_{y},
$$

where $h_{2,0}$ and $h_{0,2}$ represent the detuning with amplitude in the horizontal and vertical plane, respectively, and $h_{1,1}$ the coupling term between the two planes, while $\rho_{x}, \rho_{y}$ stand for the nonlinear invariants.

The global picture concerning the time evolution of the key beam dynamical quantities during the transverse splitting process is shown in Fig. 4. The linear chromaticity $Q_{x}^{\prime} / Q_{x}$ is decreased during the resonance crossing stage in order to reduce as much as possible the coupling between the transverse and longitudinal degrees of freedom. At the 


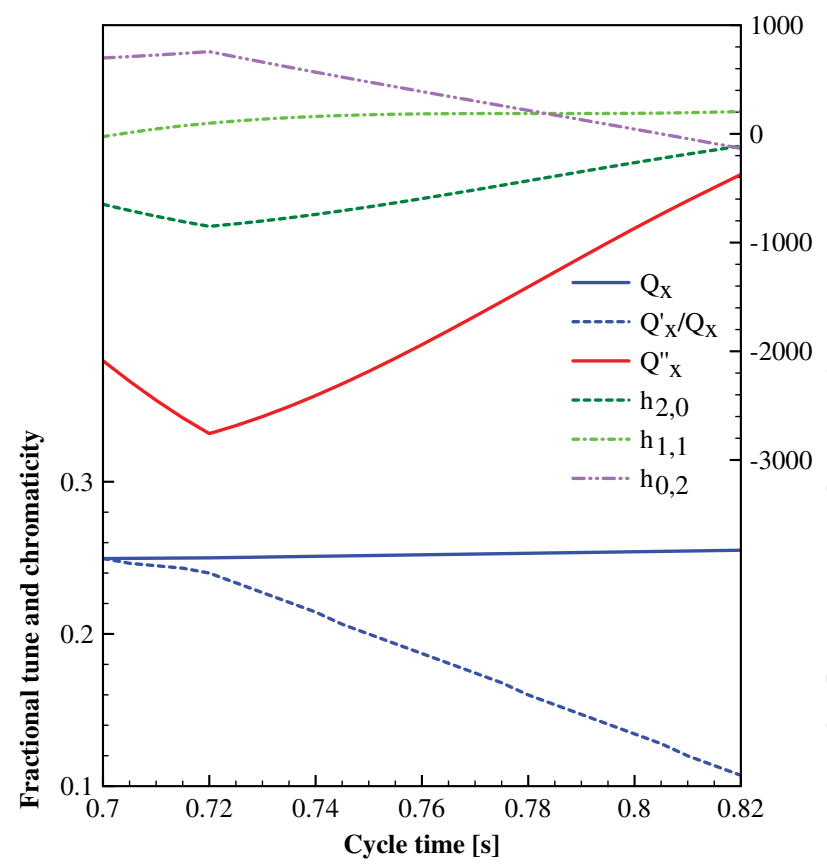

FIG. 4. Overview of the time evolution of the main physical parameters during the transverse splitting together with the horizontal tune and the linear and second-order chromaticity. The time span corresponds to the shadowed area of Fig. 2.

same time, the nonlinear coupling, represented at the lowest order by $h_{1,1}$, is essentially kept constant and small, thanks to a special circuit of seven octupoles. It is well known that the coefficients $h_{2-i, i}$ with $0 \leq i \leq 2$ are linear in the strength of the octupoles as indicated by the nonlinear, integrated gradient $K_{3}$ defined as

$$
K_{3}=\frac{1}{3 !} \frac{d^{3} B_{y}}{d x^{3}} \ell
$$

where $\ell$ stands for the magnetic length of the device. The PS main magnets feature not only the standard dipolar component, but also quadrupolar and higher-order field components generated by special windings, the so-called pole-face windings, and figure-of-eight loop [13-16]. The nonlinear coupling introduced both by sextupoles and octupoles specifically used for MTE together with that stemming from the main magnets, defined as $h_{1,1, \text { lattice }}$, can be corrected by a string of seven dedicated octupoles powered in series, according to [12]

$$
h_{1,1, \text { total }}\left(K_{3}\right)=h_{1,1, \text { lattice }}+\frac{K_{3}}{8} \sum_{i=1}^{7} \beta_{x, i} \beta_{y, i},
$$

with $\beta_{x, i}, \beta_{y, i}$ the values of the horizontal and vertical beta function at the location of the $i$ th octupole. Then, $h_{1,1, \text { total }}\left(K_{3}\right)$ is controlled in size and kept almost constant by varying the gradient $K_{3}$ of the dedicated octupoles.

The different circuits are kept to a constant value starting from $0.82 \mathrm{~s}$ from the beginning of the magnetic cycle, and during this stage, when no beam manipulation is performed, the transverse beam profile in the horizontal plane is measured to extract the dependence of the beamlets' parameters on the beam intensity.

As far as the longitudinal structure is concerned [7], a maximum of eight bunches are injected from the PS booster and then split into 16 at $3.5 \mathrm{GeV} / c$ on an intermediate plateau prior to acceleration to $14 \mathrm{GeV} / c$. At top energy a voltage reduction is applied to increase the bunch length and hence reduce the momentum spread to further decouple the longitudinal motion from the transverse during the resonance crossing. Then, just prior to extraction, the beam is completely debunched [7]. In Fig. 5, the evolution of the rf voltage during the entire magnetic cycle is shown. In order to perform the longitudinal bunch splitting, the voltage is first applied on harmonic 8, only. Then, it is decreased, while a voltage increase on harmonic 16 takes place. Typical values of the longitudinal parameters are $\epsilon_{l} \approx 0.35 \mathrm{eVs}$ and $\Delta p / p \approx 0.4 \times 10^{-3}$ (for $h=16$ ) as rms longitudinal emittance and momentum spread, respectively. The tomographic reconstruction [17] of the longitudinal phase (Fig. 5, bottom left) is plotted for the configuration corresponding to the low rf voltage after $0.68 \mathrm{~s}$ from the beginning of the magnetic cycle. The debunching gymnastic is also shown (Fig. 5, bottom right). The horizontal axis represents the bunches along the PS circumference; one turn corresponds to about $2.1 \mu \mathrm{s}$ and in this plot only half of the PS circumference is displayed. The vertical axis represents the time evolution (the origin of the axis corresponding to $0.8 \mathrm{~s}$ from the beginning of the magnetic cycle, when the rf voltage reduction starts). The debunching is clearly visible.

\section{B. PS booster beam}

The intensity of a PS beam is controlled in the PS booster (PSB) by the number of turns injected from the Linac2. However, the resulting transverse emittance is not independent of the intensity, due to the Linac2-PSB multiturn injection process (see Ref. [18] for more details).

In the experiment described in this paper the PS intensity was controlled by means of the number of turns injected into the PS booster. Nevertheless, in a few cases, the number of injected turns was kept constant, but the number of bunches delivered to the PS was varied, thus leaving some empty buckets. The effects were obtained by inhibiting the injection of bunches from one or more PSB rings. It is clear that reducing the number of bunches allows changing the total beam intensity while keeping the same transverse emittance.

The evolution of the emittance versus beam intensity can be deduced from Fig. 6, where the horizontal beam size measured with a wire scanner [19] at PS top energy is plotted as a function of beam intensity. A linear relationship between the two quantities is clearly visible. The parameters of the fit are $\left(N_{\text {total }}\right.$ is expressed in units of $10^{10}$ protons) 

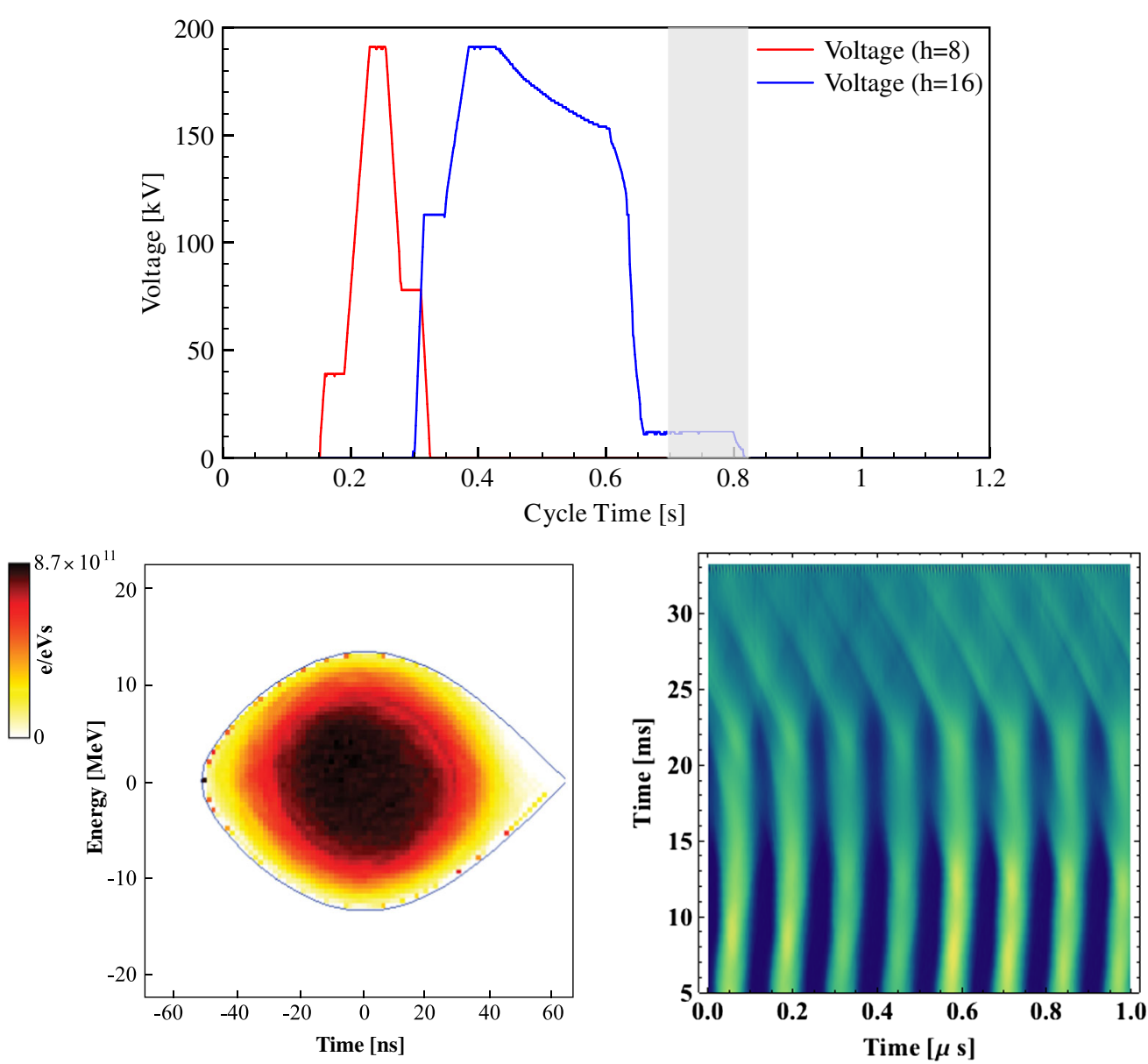

FIG. 5. Evolution of the PS $10 \mathrm{MHz}$ rf cavities voltage used for the generation of the MTE beam (upper plot). At injection, the harmonic number is $h=8$, while during the intermediate flat at $3.5 \mathrm{GeV} / c$ each bunch is split longitudinally in two and the harmonic number becomes $h=16$, which is kept constant up to beam extraction. The voltage functions used at the two harmonic numbers are plotted here. The tomographic reconstruction of the longitudinal phase (bottom left) is plotted for the configuration corresponding to the low rf voltage $0.68 \mathrm{~s}$ from the beginning of the magnetic cycle. The debunching gymnastic is also shown (bottom right). The horizontal axis represents the bunches along the PS circumference. It is recalled that one turn corresponds to $\approx 2.1 \mu \mathrm{s}$ and in this plot only half of the PS circumference is displayed. The vertical axis represents the time evolution (the origin of the axis corresponds to $0.8 \mathrm{~s}$ from the beginning of the magnetic cycle, when the rf voltage starts to be reduced). The debunching is clearly visible.

$$
\begin{aligned}
\sigma\left(N_{\text {total }}\right) & =m_{\sigma} N_{\text {total }}+b_{\sigma}, \\
m_{\sigma} & =(79553 \pm 1) \times 10^{-8} \mathrm{~mm}, \\
b_{\sigma} & =(1.91 \pm 0.03) \mathrm{mm} .
\end{aligned}
$$

For each value of the beam intensity three profile measurements are recorded and the data plotted represent the average of each series, while the error bars are the standard deviation. The value of the rms normalized emittance can be found as

$$
\epsilon_{x}^{*}\left(N_{\text {total }}\right)=\beta \gamma \frac{\left[\sigma^{2}\left(N_{\text {total }}\right)-\left(\frac{\Delta p}{p} D_{x}\right)^{2}\right]}{\beta_{x}},
$$

where $\beta, \gamma$ are the relativistic values, $\beta_{x}, D_{x}$ the beta and dispersion functions, and $\Delta p / p$ the rms momentum spread, respectively. For typical PS values of the parameters and the $\sigma$ from the measurements, $\epsilon_{x}^{*}(0)$ is different from zero. This indicates that emittance blowup is occurring, very likely at PS injection. It should be emphasized that the emittance measurements are performed on the magnetic flat top and before the resonance crossing starts and allows monitoring the beam properties before splitting. Furthermore, the few apparent outliers represent cases for which the intensity has been varied by reducing the number of bunches injected in the PS, thus keeping the transverse beam size constant. Specifically, these special cases have been obtained by starting from the highest intensity beams and then reducing by a factor of 2 or 4 the total number of injected bunches.

\section{MEASUREMENT RESULTS OF FIRST EXPERIMENTAL CAMPAIGN}

These tests were performed by injecting different proton beam intensities in the PS. Then, just prior to the resonance 


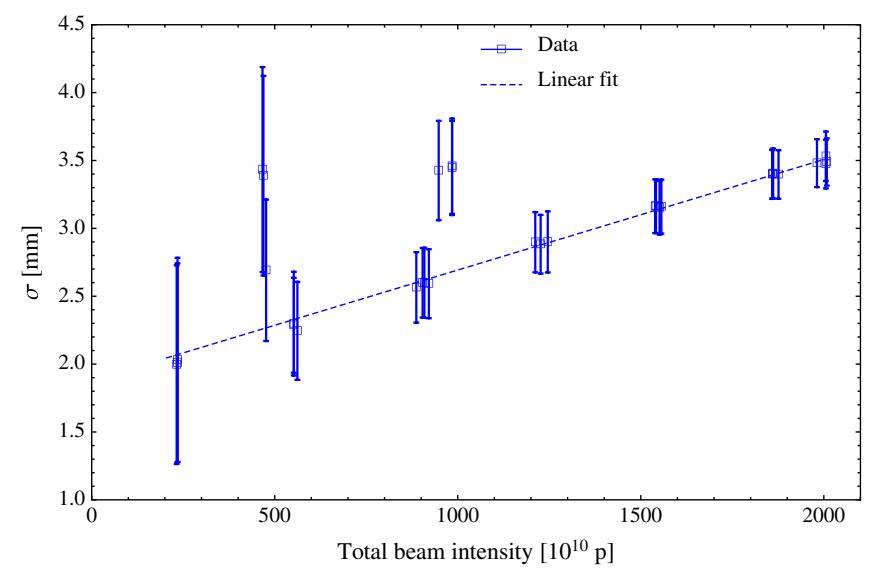

FIG. 6. Horizontal beam size before resonance crossing as a function of the total beam intensity. The linear dependence is clearly visible and is due to the multiturn injection process in the PS booster. The outliers represent the cases in which the number of injected bunches has been reduced, hence, the transverse emittance remains constant while the total beam intensity is decreased. The number of injected bunches can be controlled by inhibiting the proton beam injection into one or more PSB rings. The nonzero constant term of the linear relationship indicates that an emittance blowup is occurring, very likely at PS injection.

crossing, a horizontal beam profile was measured at $0.7 \mathrm{~s}$ from the beginning of the magnetic cycle using a wire scanner to monitor the beam size and have a cross-check of the intensity before the splitting process. Finally, at about $0.82 \mathrm{~s}$ from the beginning of the magnetic cycle, a second horizontal beam profile was measured to extract the relevant information from the beamlets after splitting. Each case, corresponding to a given intensity, was repeated three times to have minimum statistics. In Fig. 7 the

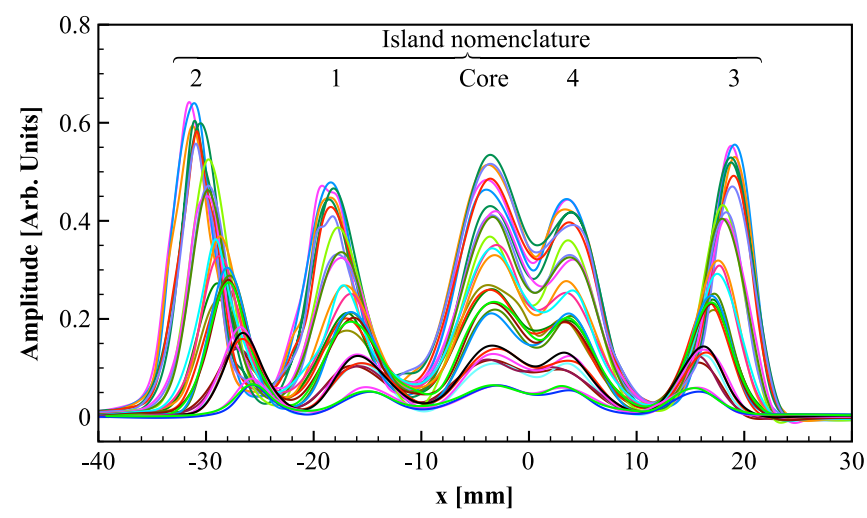

FIG. 7. Superposition of the horizontal beam profiles measured after splitting for various beam intensities. The displacement of the four outermost beamlets due to the change in the total beam intensity is clearly visible. The raw data are fitted here with splines to improve the visibility of the different profiles. The results shown in Figs. 9 and 11 have been obtained by fitting the raw data with the function of Eq. (6). superposition of the various beam profiles after splitting for the various beam intensities is shown. The five beamlets, representing the beam core, left after resonance crossing, and the particles trapped in the stable islands, are clearly visible as five Gaussian-like profiles. It is also clearly seen that, while the core is unaffected, in terms of mean position and sigma, by the different total beam intensity, the beamlets are indeed showing some dependence, as their position is changing with the total beam intensity. A more quantitative analysis is performed by fitting five Gaussian distribution functions and studying the behavior of average and sigma as a function of intensity. The fitting function used for the data analysis is

$G(x)=\frac{N_{c}}{\sqrt{2 \pi} \sigma_{c}} e^{-\left(\left(x-\mu_{c}\right)^{2} / 2 \sigma_{c}^{2}\right)}+N_{\mathrm{isl}} \sum_{i=1}^{4} \frac{1}{\sqrt{2 \pi} \sigma_{i}} e^{-\left(\left(x-\mu_{i}\right)^{2} / 2 \sigma_{i}^{2}\right)}$,

where $N_{c}, \mu_{c}, \sigma_{c}$ represent the number of particles, the mean value, and the sigma of the beam core. For the islands $\mu_{i}, \sigma_{i}$, with $1 \leq i \leq 4$, stand for their mean values and sigmas, while $N_{\text {isl }}$ represent the number of particles trapped in each island. Note that Eq. (6) provides a tenparameter fit function to the measured horizontal beam profile. It is also clear that in this model the intensity of each island is constrained to be identical. This is a direct consequence of the splitting process that creates, by definition, four equally populated beamlets. In addition, each measured transverse profile is the result of a slow passage of the wire scanner, slower than the beam revolution time, through the protons. Therefore, the final result necessarily represents an average situation for the beamlets.

In Fig. 8, $\mu_{c}$ and $\sigma_{c}$ are plotted as a function of the total beam intensity. No clear trend is visible, showing that the physical parameters of the beam left in the core are intensity independent. Another feature is the increase of the error bars for smaller intensities, which is more relevant for the beam sigma rather than its average position. This is due to the fact that for lower intensity the beamlets are less separated, which affects the accuracy of the reconstruction of the five Gaussian functions. Furthermore, it has been decided not to change the wire scanner photomultiplier gain during the measurements, which would have been a possible mitigation measure to improve the signal-to-noise ratio for low beam intensities. In fact, the detection of the secondary particles emitted by the wire is made by two scintillators connected to two photomultipliers located downstream of the instrument, on each side of the vacuum chamber, approximately in the median plane. The response of the two photomultipliers is not exactly the same, which could induce some left/right asymmetry with an impact on the reconstructed properties of the beamlets. A change of gain during the measurement might alter the profile symmetry as a function of the total beam intensity, thus making the analysis more problematic. For this reason it has been 

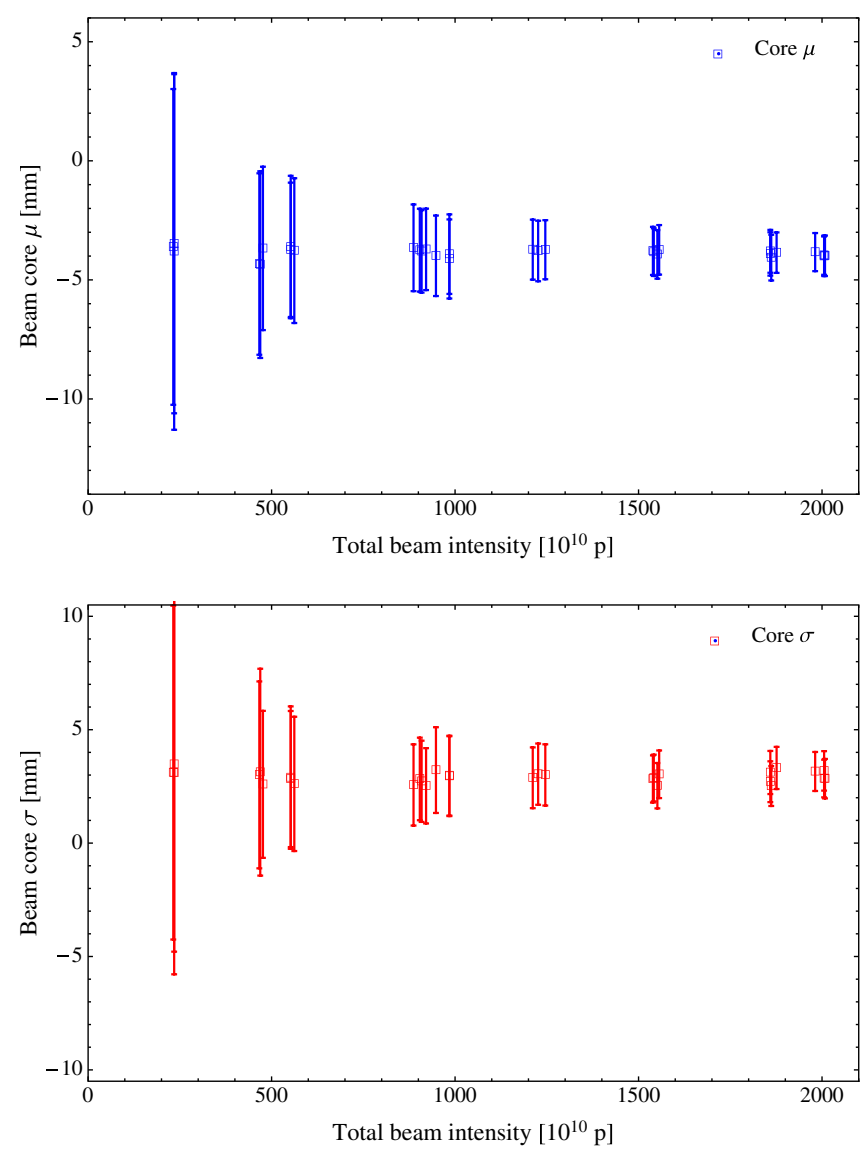

FIG. 8. Beam core position $\mu_{c}$ (upper) and sigma $\sigma_{c}$ (lower), from the fit using Eq. (6) of the data shown in Fig. 7, vs total beam intensity. The error bars represent the error associated with the fitting procedure. The larger errors for lower total beam intensities reflect the difficulty of representing the close-by peaks with five Gaussian distribution functions, and also the effect of the constant gain used for the wire scanner throughout the whole measurement campaign. Neither $\mu_{c}$ nor $\sigma_{c}$ feature any dependence on the total beam intensity.

decided to accept some degradation of the accuracy in the profile measurement at low intensity, which would be in any case negligible for the purpose of identifying the beamlets' position, but to ensure that the potentially induced profile asymmetry does not depend on the beam intensity. Nevertheless, the photomultipliers were always operating in a linear regime. It is also clear that $\mu$ is less affected than $\sigma$.

In Fig. 9, the position $\mu_{i}$ of the peak of the four Gaussian functions is plotted as a function of the total intensity. A clear linear trend is visible and the fit parameters are reported in Table I together with the associated fit errors. The slopes of these lines are different, partly due to the projection effects of the islands' position from the horizontal phase space to real space. The smaller emittance obtained for lower intensity beams generates a lower fraction of trapped particles and hence a lower quality for the

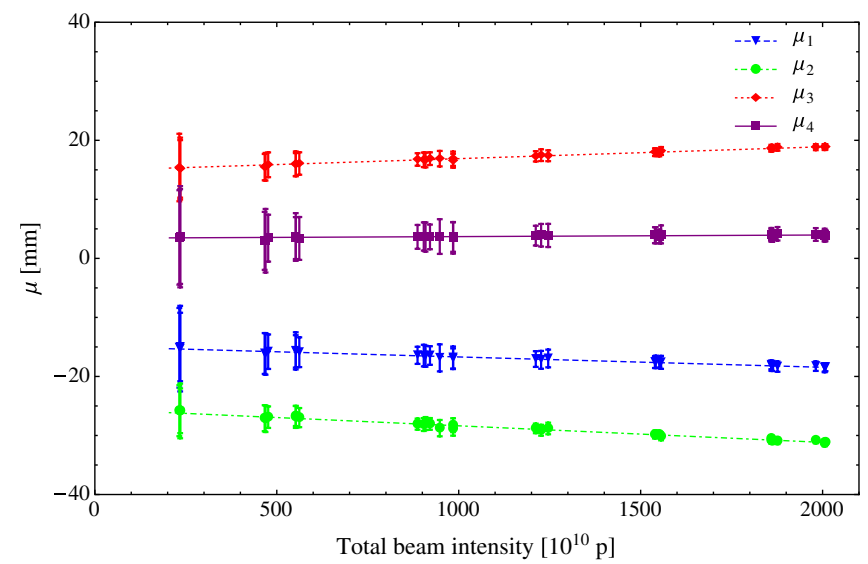

FIG. 9. Beamlets' position $\mu_{i}$, from the fit using Eq. (6) of the data shown in Fig. 7, vs total beam intensity. The error bars represent the error associated with the fitting procedure. The larger errors for lower total beam intensities reflect the difficulty of representing the close-by peaks with five Gaussian distribution functions, and also the effect of the constant gain used for the wire scanner throughout the whole measurement campaign. The excellent quality of the linear fit of $\mu_{i}$ vs intensity is clearly visible.

Gaussian fit. This explains the increased size of the error bars towards lower intensities, together with the choice of keeping the wire scanner gain constant. The fraction of trapped particles is defined as

$$
\tau=\frac{N_{\text {isl }}}{N_{\text {initial }}}
$$

where $N_{\text {initial }}$ refers to the value of the parameter of the Gaussian best fitting the measured beam profile before the transverse splitting gymnastics. The dependence of $\tau$ on the beam intensity is shown in Fig. 10. No particular trend is visible, with $\tau$ ranging in the interval $17 \%-21 \%$. Parenthetically, during these tests a factor of 3 in emittance variation for the interval of total beam intensity probed produces a variation of only $23 \%$ in $\tau$.

Another interesting feature of the process is that the $\sigma_{i}$ for the beamlets are intensity independent, as shown in Fig. 11, and are rather independent of the specific island considered. In this case the quality of the fit decreases with the lower beam intensities as well.

TABLE I. Linear fit results of the islands' position $\mu_{i}$ as a function of total beam intensity ( $N_{\text {total }}$ in units of $10^{10}$ protons) obtained from the data plotted in Fig. 9.

\begin{tabular}{lcc}
\hline \hline Island & Slope $(\mathrm{mm})$ & Intercept $(\mathrm{mm})$ \\
\hline 1 & $(-18 \pm 5) \times 10^{-4}$ & $-14.9 \pm 0.9$ \\
2 & $(-28 \pm 3) \times 10^{-4}$ & $-25.4 \pm 0.6$ \\
3 & $(20 \pm 4) \times 10^{-4}$ & $14.8 \pm 0.6$ \\
4 & $(3 \pm 7) \times 10^{-4}$ & $3 \pm 1$ \\
\hline \hline
\end{tabular}




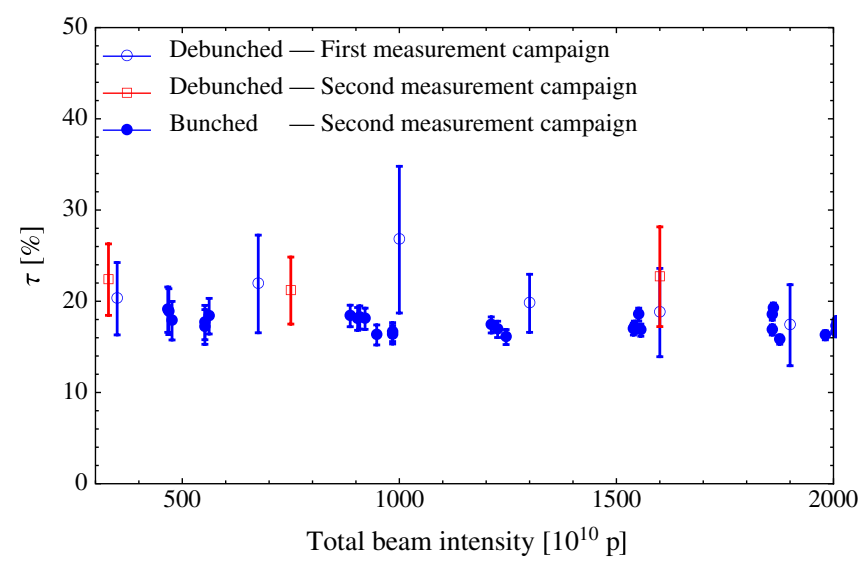

FIG. 10. Evolution of the trapping fraction $\tau$ as a function of the total beam intensity. The error bars are derived from the errors associated to the fit procedure for obtaining the parameters $N_{\text {isl }}, N_{\text {initial }}$. The data reported are those from the first and second measurement campaigns. No meaningful difference between the two campaigns or the type of longitudinal structure is visible.

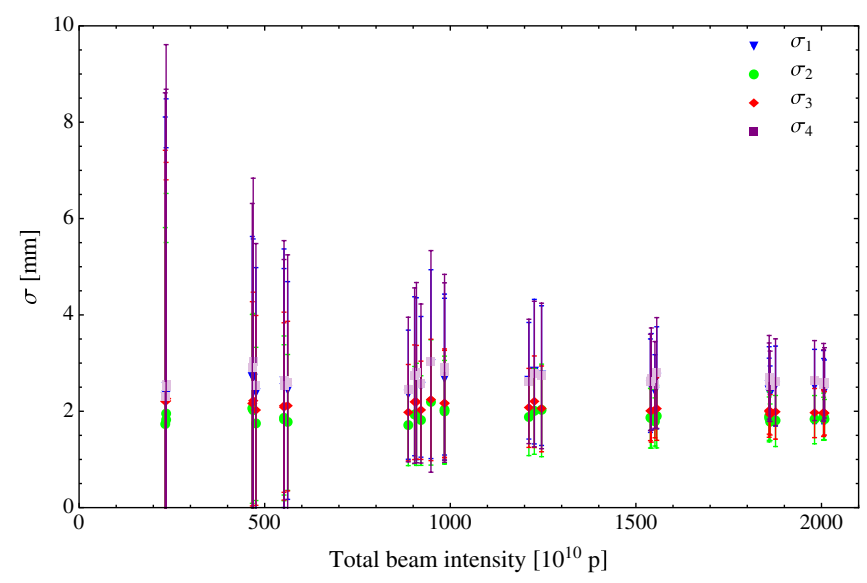

FIG. 11. Beamlets' sigma $\sigma_{i}$, from the fit using Eq. (6) of the data shown in Fig. 7, vs total beam intensity. Also in this case the error bars are determined from the fitting procedure and feature a clear degradation towards low intensities. Nevertheless, no relevant difference of beam size for the four beamlets is visible. Furthermore, no dependence on the total beam intensity can be detected.

\section{DISCUSSION OF EXPERIMENTAL RESULTS OF FIRST EXPERIMENTAL CAMPAIGN}

The analysis of the measurements indicates that the physical characteristics of the beam core are essentially unaffected by the change in total beam intensity. On the other hand, the mean position of the beamlets is clearly affected, but their size is not sensitive to the total beam intensity.

Based on these observations, our conjecture is that the effect of the total beam intensity is equivalent to producing primarily a shift of the linear tune. Furthermore, as the initial system, in a regime of negligible space charge effects exhibits a negative detuning with amplitude, such an intensity-dependent tune shift should be positive. If, instead of a simple shift of the linear tune a nonlinear effect was assumed, then it should have generated not only a change of the beamlets' position, via the change of detuning with amplitude, but also a change of the islands' size and shape. In turn, this effect should have been observed on the measured sigmas, either of the beam core or of the beamlets. This effect, however, is not visible in the measured data. Moreover, while the beam core would possibly not be too sensitive to strong nonlinear effects, as they tend to decrease rapidly towards the origin of phase space, the beamlets are certainly in an ideal condition to probe nonlinear effects. Nonetheless, as already mentioned, no sizeable change in $\sigma_{i}$ was observed.

It is clear that the tune shift should be partly generated by the direct space charge effects between the five beamlets, and partly by the image currents flowing in the vacuum chamber. Here, no consideration to the effects that depend on the finite resistivity of the vacuum chamber is made. In the PS machine, only variants of elliptical shapes are used for the cross section of the vacuum chambers. For the time being, however, no attempt is made to estimate these two effects separately, and only the global effect is considered in the following analysis. Nevertheless, it is already possible to conclude that, as direct space charge effects in the transverse planes always produce a defocusing effect, so that the tune shift is negative, the experimental observations, according to which the net effect is a positive horizontal tune shift, indicate that the contribution from image currents is larger than that of the direct effects.

The experimental observations have provided data about the islands positions as a function of the total beam intensity (see Table I). The intercept of the linear fit provides the beamlets' position for zero intensity, while the numerical model of the PS machine [20] can be used to estimate the position of the fixed points of the fourth-order resonance as a function of the linear tune. Such a model have been built by reconstructing the nonlinear components of the magnetic field of the PS main magnets by means of fitting sextupolar and octupolar multipoles to measured values of the horizontal and vertical tunes as a function of the momentum offset. In Fig. 12 the fixed points' position, corresponding to the beamlets' position, computed numerically has been plotted as a function of the fractional part of the horizontal linear tune. The four fixed points feature a linear dependence on the fractional tune and straight lines can be fitted to the numerical data. The fit parameters are listed in Table II.

It should be stressed that the closed orbit at the location of the wire scanner is not zero, but about $-4 \mathrm{~mm}$. This can be inferred from the results of the fit of the beam core. Therefore, in the comparison between the measured beamlets' position and those of the numerical model, such a shift must be taken into account. By combining the functions 


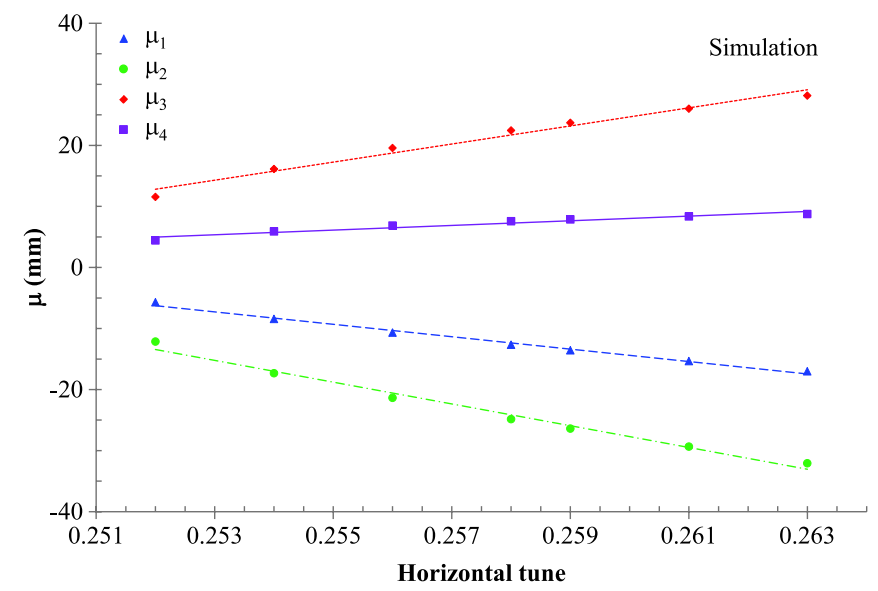

FIG. 12. The position of the fixed points as a function of the horizontal tune is plotted. The numerical model of the PS ring has been used. Such a model has been built starting from measurements of tunes as a function of momentum offset.

expressing the beamlets' position vs total beam intensity and vs linear tune it is possible to find the relationship giving the tune shift due to the beam intensity that would be needed to reproduce the observed beamlets' displacements, or

$$
Q_{x}^{i}\left(N_{\text {total }}\right)=m_{i, Q_{x}} N_{\text {total }}+Q_{x, 0}^{i},
$$

where $i$ indicates which beamlet is used for determining the linear relation between tune and intensity. In Table III the parameters $m_{i, Q_{x}}$ and $Q_{x, 0}^{i}$ are listed, together with the associated errors. A rather good agreement between the fitted parameters for three out of the four beamlets is found. The final result is obtained by taking the weighted average of the four relationships and gives

TABLE II. Linear fit results of the islands' position $\mu_{i}$ as a function of the fractional part of the tune from the simulation data plotted in Fig. 12.

\begin{tabular}{lrc}
\hline \hline Island & Slope $(\mathrm{mm})$ & Intercept $(\mathrm{mm})$ \\
\hline 1 & $-1016 \pm 41$ & $250 \pm 11$ \\
2 & $-1783 \pm 96$ & $436 \pm 25$ \\
3 & $1480 \pm 96$ & $-360 \pm 25$ \\
4 & $380 \pm 42$ & $-91 \pm 11$ \\
\hline \hline
\end{tabular}

TABLE III. Linear relationships between fractional tune and total beam intensity ( $N_{\text {total }}$ in units of $10^{10}$ protons) as derived from the experimental and numerical data reported in Tables I and II.

\begin{tabular}{lcc}
\hline \hline Island & $m_{i, Q_{x}}(\mathrm{~mm})$ & $Q_{x, 0}^{i}$ \\
\hline 1 & $(172 \pm 7) \times 10^{-8}$ & $0.257 \pm 0.023$ \\
2 & $(157 \pm 8) \times 10^{-8}$ & $0.257 \pm 0.029$ \\
3 & $(136 \pm 9) \times 10^{-8}$ & $0.256 \pm 0.036$ \\
4 & $(71 \pm 8) \times 10^{-8}$ & $0.258 \pm 0.066$ \\
\hline \hline
\end{tabular}

$$
Q_{x}\left(N_{\text {total }}\right)=m_{Q_{x}} N_{\text {total }}+Q_{x, 0},
$$

or

$$
\Delta Q_{x}\left(N_{\text {total }}\right)=m_{Q_{x}} N_{\text {total }},
$$

where $\Delta Q_{x}$ represents the tune shift as a function of the total beam current at the end of the splitting process and

$$
m_{Q_{x}}=(15 \pm 4) \times 10^{-7}, \quad Q_{x, 0}=0.257 \pm 0.033 .
$$

\section{MEASUREMENT RESULTS OF SECOND EXPERIMENTAL CAMPAIGN}

A second experimental campaign has been undertaken in 2012 to assess a more specific point, namely, the dependence of the beamlets' position on the longitudinal beam structure. Indeed, the longitudinal beam distribution has an impact on the space charge effects and it could also affect the position of the beamlets after splitting. To this aim, two extreme cases have been considered, i.e., the bunched structure with 16 bunches and the same parameters as in the 2011 experimental sessions, and a debunched structure. This is achieved by reducing adiabatically the rf voltage prior to the resonance crossing. It is worth mentioning that the bunching factor, defined as

$$
\frac{1}{B}=\frac{3 \pi R}{L M},
$$

where the longitudinal beam distribution is assumed to be parabolic and $R, L$, and $M$ are the PS radius $(100 \mathrm{~m})$, the total bunch length $(\approx 37 \mathrm{~m})$, and number of bunches (16), respectively, is of the order of 1.6 for the cases under consideration. This means that the impact of the longitudinal space charge forces is in principle non-negligible.

The aim of these additional studies has been to perform a direct comparison of the beamlet's position as a function of the total beam intensity. The wire scanner has been used to record the final position of the beamlets and the fitting procedure already described has been applied to the data with the two longitudinal beam distributions. The new campaign has been carried out using a wire scanner in a different section of the PS ring with respect to the one used during the 2010 studies: in Fig. 3 (right) the orientation of the islands at the location of the wire scanner used in 2012 is visible, as well as the phase space topology corresponding to the 2010 configuration (left). In Fig. 13 (upper plot) the final beamlets' position as a function of the total beam intensity is shown for both beams. The linear dependence on the intensity is confirmed also in this case, while no difference in the behavior is noticed for the two longitudinal structures. Direct inspection of the numerical values of the linear fit parameters reported in Table IV confirms that they are the same within the measurement errors.

Similarly to what has been already mentioned, neither the $\sigma$ of the beamlets (see Fig. 13 lower plot) nor the trapping 

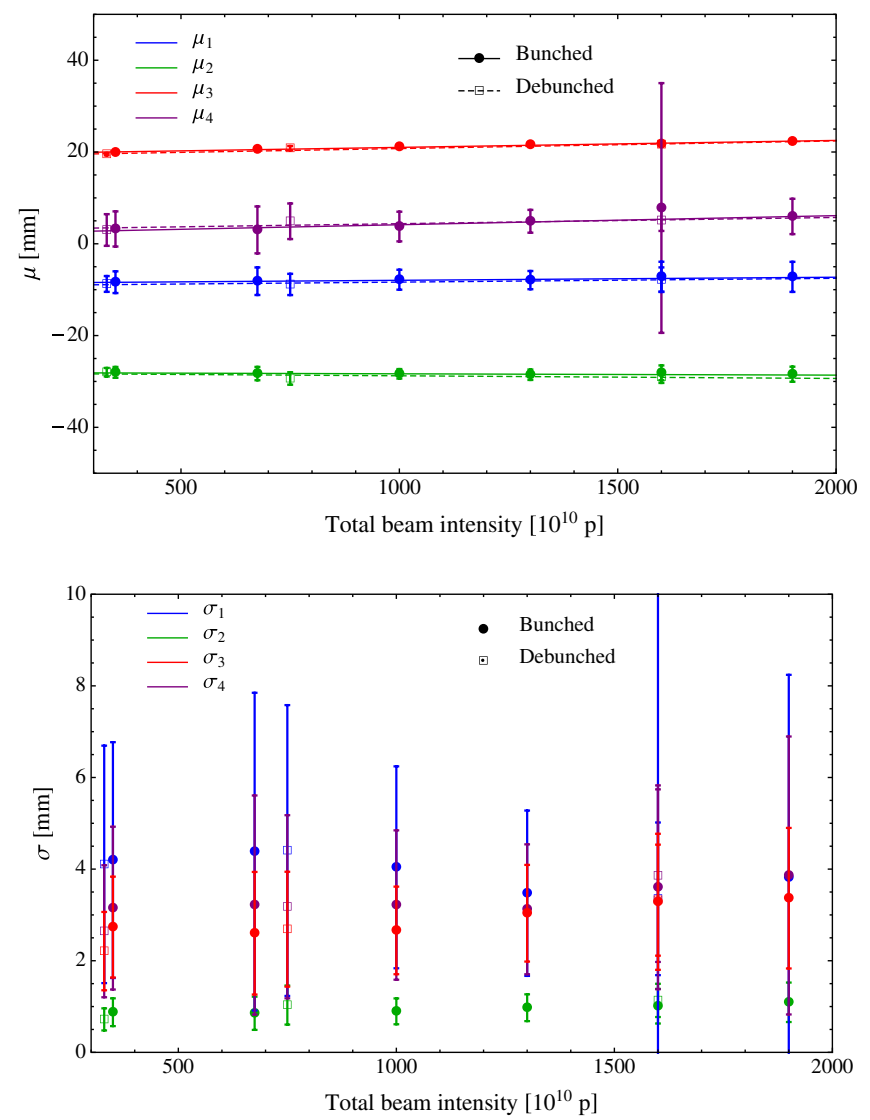

FIG. 13. Beamlets' position $\mu_{i}$, from the fit using Eq. (6) of the data collected during the second experimental campaign, vs total beam intensity (upper plot). The data from the debunched as well as bunched beam are shown. The excellent quality of the linear fit of $\mu_{i}$ vs intensity is clearly visible. The differences between the two beam types seems negligible. The beamlets' size $\sigma_{i}$ vs total beam intensity is also shown (lower plot) and no clear dependence on the beam intensity is visible.

fraction (see Fig. 10) depend critically on the total beam intensity for either type of beams.

The evolution of the beamlets' position during the trapping and separation process has also been probed, in view of searching for meaningful differences that could be associated with the different longitudinal structure. In this case, profile measurements have been taken during the splitting process for both debunched and bunched beams. Fitting five Gaussian distributions to the measured profiles corresponding to early stages of the trapping process can be really hard. This is because the beamlets can be very close to the central core and it might be extremely challenging to disentangle the various peaks resulting from the projection from phase space to the physical space. Therefore, for this analysis, the information has been taken only from the two outermost beamlets. In Fig. 14 the plots of the beamlets' position $\mu_{i}$ vs horizontal tune are shown for the beamlet number 2 (upper plot) and 3 (lower plot) and the two types of beams. Parabolic fit functions of the form

$\mu_{i}\left(\nu_{x}\right)=\lambda_{0, i}+\lambda_{1, i}\left(\nu_{x}-0.258\right)+\lambda_{2, i}\left(\nu_{x}-0.258\right)^{2}$,

with $\nu_{x}$ the fractional part of the horizontal tune, are also overlaid and the agreement is remarkable. The initial assumption has been to consider different sets of parameters $\lambda_{j, i}$ for the different intensities and beam types. The results of this first hypothesis are visible in Fig. 15 where the fit parameters derived from $\mu_{3}$ are shown in the case of an unconstrained fit. Of course, it is apparent that only the constant term shows a non-negligible dependence on the total beam intensity. Furthermore, the resulting fit parameters are hardly sensitive to the longitudinal beam structure. For this reason, a second approach has been used, based on a global fit, in which the linear and quadratic terms are assumed to be independent on intensity and longitudinal beam structure, while the constant term depends linearly on the beam intensity, only. The resulting global fit parameters are also shown in Fig. 15 and the obtained fit functions are overlaid to the beamlets' position evolution in Fig. 14 from which the excellent agreement with the experimental data is visible. The fact that the fit curves corresponding to the three different beam intensities are almost superimposed in the case of $\mu_{2}$ reflects the very small value of the slope of the straight line fitting $\mu_{2}$ as a function of intensity (see Table IV).

It is worth emphasizing that the measurements of the transverse beam profiles used to evaluate the displacement

TABLE IV. Comparison of the fit results of the islands' position $\mu_{i}$ as a function of total beam intensity ( $N_{\text {total }}$ in units of $10^{10}$ protons) obtained from the second experimental campaign for the debunched and bunched beams. The two sets of values are compatible within the error bars. These values cannot be directly compared with those of Table I as they refer to a different location in the PS ring.

\begin{tabular}{lcccr}
\hline \hline & \multicolumn{2}{c}{ Debunched beam } & \multicolumn{2}{c}{ Bunched beam } \\
Island & Slope $(\mathrm{mm})$ & Intercept $(\mathrm{mm})$ & Slope $(\mathrm{mm})$ & Intercept $(\mathrm{mm})$ \\
\hline 1 & $(8 \pm 1) \times 10^{-4}$ & $-8.7 \pm 0.2$ & $(8 \pm 4) \times 10^{-4}$ & $-9.2 \pm 0.4$ \\
2 & $(-2 \pm 1) \times 10^{-4}$ & $-28.1 \pm 0.2$ & $(-6 \pm 9) \times 10^{-4}$ & $-28.2 \pm 0.9$ \\
3 & $(15 \pm 1) \times 10^{-4}$ & $19.5 \pm 0.1$ & $(15 \pm 5) \times 10^{-4}$ & $19.3 \pm 0.5$ \\
4 & $(27 \pm 9) \times 10^{-4}$ & $2 \pm 1$ & $(14 \pm 11) \times 10^{-4}$ & $3 \pm 1$ \\
\hline \hline
\end{tabular}



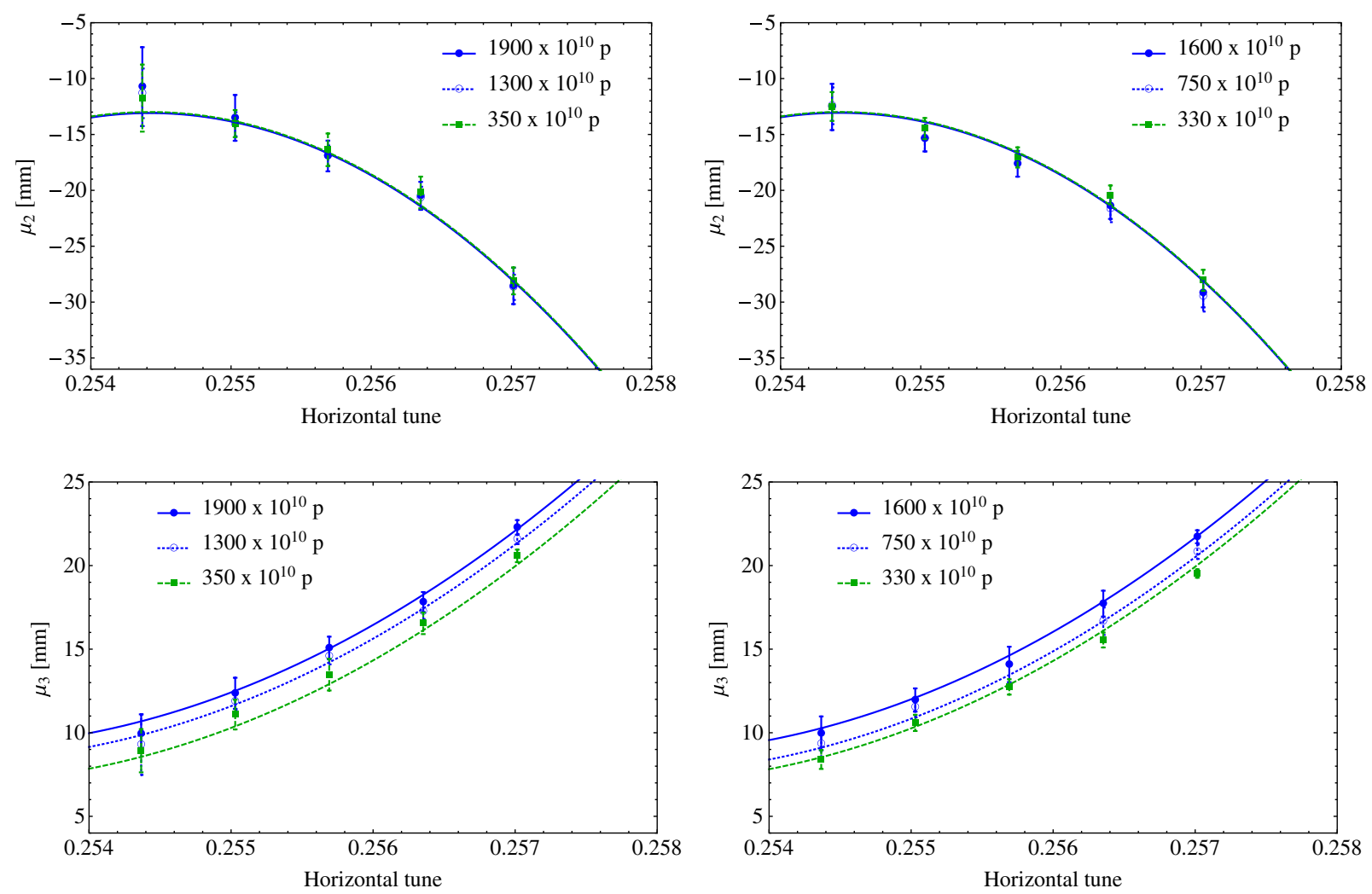

FIG. 14. Beamlets' position $\mu_{2}$ (upper) and $\mu_{3}$ (lower) of the data collected during the second experimental campaign vs horizontal tune evolution for three values of the beam intensity. The data from the debunched (left) as well as bunched beam (right) are shown. The quadratic fit functions shown in the plots represent the global fit discussed in the text. The excellent agreement is clearly visible.

of the beamlets in the transverse plane took place in a time window ranging from $0.72 \mathrm{~s}$ to $0.76 \mathrm{~s}$, hence earlier than the measurements performed during the first campaign $(0.82 \mathrm{~s})$. Indeed, the time functions of the nonlinear elements shown in Fig. 2 have been shifted by $-0.06 \mathrm{~s}$ for the second measurement campaign. During the wire scanner measurements the sextupoles and octupoles were changing in strength, in addition to the linear tune, resulting in a nonlinear variation of the beamlets' position as a function of the horizontal tune. Starting from $0.76 \mathrm{~s}$ the PS ring was again in a static configuration. On the other hand, the linear dependence of the beamlets' position seen in Fig. 12 is the result of a static configuration of the PS machine in terms of strength of the nonlinear elements used for the trapping process.

All these observations allow one to conclude that the longitudinal beam structure is not affecting the final beamlets' position in spite of a value of the bunching factor sufficiently different from 1 . More than this, the way the beamlets are moving apart during the trapping process depends on the total beam intensity only via the constant term of the parabolic dependence on the tune value, i.e., as

$$
\lambda_{0, i}=\bar{\lambda}_{0, i}+\hat{\lambda}_{0, i} N_{\text {total }}
$$

The linear and quadratic terms of the parabolic fit are largely independent from the beam intensity, thus indicating no presence of nonlinear effects due to space charge. This confirms previous observations that the net overall effect of the space charge forces is a plain shift of the single-particle tune. In fact, by assuming that $m_{Q_{x}}$ is small enough to justify neglecting second-order effects, which is the assumption required to match the experimental observations otherwise, also $\lambda_{1, i}$ and $\lambda_{2, i}$ would show some dependence on $N_{\text {total }}$, then by replacing Eq. (14) in Eq. (13) one obtains that

$$
m_{Q_{x}, i}=-\frac{\hat{\lambda}_{0, i}}{\lambda_{1, i}}
$$

As a last comment, we would like to point out that the dependence of beamlets' position with tune is very smooth and no change is observed when reaching higher amplitudes. This implies that, with the current data, it is not possible to assess whether image effects have a role in the observed phenomena, or the shift of $\mu_{i}$ with total beam intensity is the result of the direct space charge forces only. In fact, one could imagine observing a change of the dependence of the beamlets' position at some threshold amplitude, at which the image effects would start dominating over the direct space charge; however, this hypothesis is not supported by the data collected. 

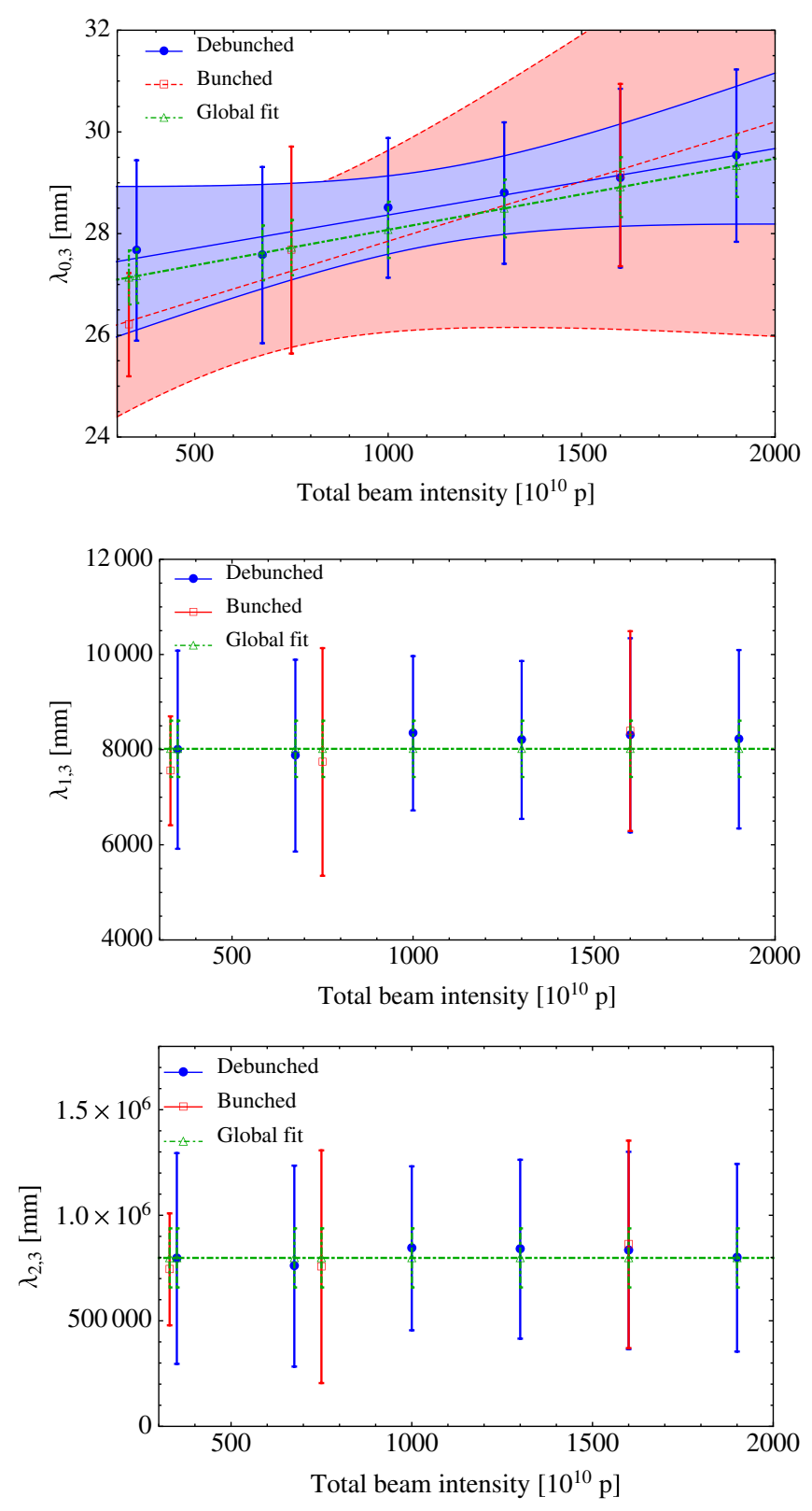

FIG. 15. Dependence of the quadratic fit parameters $\lambda_{i, 3}$ on the beam intensity. The unconstrained fit parameters together with the global one (green lines) are shown. The shaded areas in the plot of $\lambda_{0,3}$ represent the prediction bands around the estimated linear fit lines at $95 \%$ confidence level. The straight line obtained for the global fit is in the intersection of the bands for the debunched and bunched beams predictions.

\section{CONCLUSIONS}

In this paper, the first measurements of intensitydependent effects for transversely split beams have been reported. These observations were made during the commissioning period of the CERN PS MTE beams. According to these measurements, the final position of the beamlets is a linear function of the total beam intensity. As the other physical properties of the four beamlets and the beam core do not show any measurable change with the total intensity, it has been assumed that the observed effects can be interpreted in terms of a positive shift of the linear tune. Based on the data collected the tune shift has been evaluated.

Transversely split beams are a relatively new possibility and open up new regimes and phenomena in beam dynamics, in particular, in the domain of space charge effects. The observed effect, of course, is the superposition of two different components, namely, the direct space charge between the beamlets and the beam core, and the beamlets' interaction with the image charges on the vacuum chamber walls. Any consideration on the finite resistivity of the vacuum chamber would add an additional degree of complexity to the problem discussed in this paper. Furthermore, it is worth emphasizing that the transversely split beams might open up also new possibilities for high-sensitivity impedance measurements. This is because of the intrinsic large, and in any case controllable, amplitude of the beamlets that can probe regions of the machine aperture that are normally not accessible by standard beams in a stable fashion, i.e., without inducing large-amplitude betatronic oscillations.

In this article only the total net effect is evaluated, but it is clear that in the future it will be useful to perform a thorough theoretical analysis to attempt to estimate these two effects separately. A potential approach could be the use of simple analytical models, describing space charge effects in conjunction with external magnetic nonlinearities (see, e.g., Refs. [21,22]).

The measurements and the subsequent analysis and interpretation are aimed at understanding the final stage of the splitting process, namely, the final position of the beamlets. The splitting process proper might also be affected by space charge effects. Nevertheless, the observations reported in this paper (beamlets' characteristics and fraction of total beam intensity trapped in the beamlets) seem to indicate that intensity-dependent effects have a negligible impact on the splitting stage. This observation has been confirmed by the second measurements campaign, which has shown that the longitudinal beam distribution is not affecting the transverse beam position that seems to depend only on the total beam intensity. Furthermore, measurements of the beamlets' position during the resonance crossing stage have revealed that the evolution is a expressed by a parabolic function of the tune, which is shifted with intensity only via a change in the constant term.

Finally, it is well known that resonance crossing features some asymmetries, depending on the direction of crossing. In this paper it is confirmed that this is the case for transversely split beams in the presence of intensity-dependent effects. Indeed, for the PS standard case, with the horizontal tune crossing from below the fourth-order resonance, the detuning with amplitude, generated by external magnetic 
nonlinearities, is negative. The intensity-dependent effects have been observed to separate the beamlets. Therefore, this can be interpreted as being equivalent to a positive, intensity-dependent tune shift. We would like to stress that the opposite would occur for the case of a reverse direction of resonance crossing.

\section{ACKNOWLEDGMENTS}

We would like to thank A. Bazzani and G. Franchetti for interesting discussions on this topic. Several inspiring exchanges of ideas with E. Métral and the excellent comments of E. McIntosh on the original manuscript are warmly acknowledged.

[1] R. Cappi and M. Giovannozzi, Phys. Rev. Lett. 88, 104801 (2002).

[2] R. Cappi and M. Giovannozzi, Phys. Rev. ST Accel. Beams 7, 024001 (2004).

[3] C. Bovet, D. Fiander, L. Henny, A. Krusche, and G. Plass, IEEE Trans. Nucl. Sci. 20, 438 (1973).

[4] M. Giovannozzi, M. Barnes, O. Berrig, A. Beuret, J. Borburgh, J.-P. Bourquin, R. Brown, J.-P. Burnet, F. Caspers, J.-M. Cravero, T. Dobers, T. Fowler, S. Gilardoni, M. Hourican, W. Kalbreier, T. Kroyer, F. Di Maio, M. Martini, V. Mertens, E. Métral, K.-D. Metzmacher, C. Rossi, J.-P. Royer, L. Sermeus, R. Steerenberg, G. Villiger, and T. Zickler, Report No. CERN-2006-011, 2006.

[5] S. Gilardoni, M. Giovannozzi, M. Martini, E. Métral, P. Scaramuzzi, R. Steerenberg, and A. S. Müller, Phys. Rev. ST Accel. Beams 9, 104001 (2006).

[6] A. Franchi, S. Gilardoni, and M. Giovannozzi, Phys. Rev. ST Accel. Beams 12, 014001 (2009).

[7] E. Benedetto, A. Blas, T. Bohl, S. Cettour Cave, K. Cornelis, D. Cotte, H. Damerau, M. Delrieux, J. Fleuret, T. Fowler, F. Follin, A. Franchi, P. Freyermuth, H. Genoud, S. Gilardoni, M. Giovannozzi, S. Hancock, O. Hans, Y. Le Borgne, D. Manglunki, E. Matli, E. Métral, G. Métral, M. Newman, L. Pereira, F. Peters, Y. Riva, F. Roncarolo, L. Sermeus, R. Steerenberg, B. Vandorpe, and J. Wenninger, in Proceedings of First International Particle Accelerator Conference, edited by A. Noda, C. Petit-Jean-Genaz, V. Schaa, T. Shirai, and A. Shirakawa (Institute of Physics, London, England, 2010), p. 3619.

[8] S. Gilardoni, D. Manglunki, J.-P. Burnet, C. Carli, M. Chanel, R. Garoby, S. Gilardoni, M. Giovannozzi, S. Hancock, H. Haseroth, K. Hübner, D. Küchler, J. Lewis, A. Lombardi, D. Manglunki, M. Martini, S. Maury, E. Métral, D. Möhl, G. Plass, L. Rinolfi, R. Scrivens, R. Steerenberg, C. Steinbach, M. Vretenar, and T. Zickler, Report No. CERN-2011-004, 2011.

[9] M. Giovannozzi and J. Morel, Phys. Rev. ST Accel. Beams 10, 034001 (2007).

[10] M. Giovannozzi, M. George, and A. Franchi, in Proceedings of First International Particle Accelerator Conference, edited by A. Noda, C. Petit-Jean-Genaz, V. Schaa, T. Shirai, and A. Shirakawa (Institute of Physics, London, England, 2010), p. 4710.

[11] S. Gilardoni and M. Giovannozzi, in Proceedings of Second International Particle Accelerator Conference, edited by A. Noda and C. Petit-Jean-Genaz (Institute of Physics, London, England, 2010), p. 631.

[12] A. Bazzani, E. Todesco, G. Turchetti, and G. Servizi, Report No. CERN-94-02, 1994.

[13] R. Gouiran, Report No. CERN MPS/SM/Note 75-21, 1975.

[14] R. Gouiran, Report No. CERN MPS/SM/Note 74-25, 1974.

[15] R. Gouiran, Report No. CERN PS/SM/76-1, 1976.

[16] J. Gruber, Report No. CERN MPS/SM/Spec 75-8 1975.

[17] S. Hancock, M. Lindroos, E. McIntosh, and M. Metcalf, Comput. Phys. Commun. 118, 61 (1999).

[18] P. D. V. van der Stok, Report No. CERN-PS-BR-81-28, 1981.

[19] Ch. Steinbach and M. van Rooij, IEEE Trans. Nucl. Sci. 32, 1920 (1985).

[20] R. Cappi, M. Giovannozzi, M. Martini, E. Métral, G. Métral, R. Steerenberg, and A.-S. Müller, in 2003 Particle Accelerator Conference, edited by J. Chew, P. Lucas, and S. Webber, (IEEE Computer Society Press, Piscataway, NJ, 2003), p. 2913.

[21] L. Bongini, A. Bazzani, G. Turchetti, and I. Hofmann, Phys. Rev. ST Accel. Beams 4, 114201 (2001).

[22] C. Benedetti and G. Turchetti, Phys. Lett. A 340, 461 (2005). 